\title{
Nanohole Array-directed Trapping of Mammalian Mitochondria Enabling Single Organelle Analysis
}

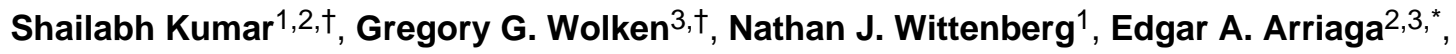 \\ and Sang-Hyun $\mathrm{Oh}^{1,2, *}$ \\ ${ }^{1}$ Department of Electrical and Computer Engineering, University of Minnesota, Minneapolis, MN \\ 55455, United States \\ ${ }^{2}$ Department of Biomedical Engineering, University of Minnesota, Minneapolis, MN 55455, United \\ States \\ ${ }^{3}$ Department of Chemistry, University of Minnesota, Minneapolis, MN 55455, United States
}

\begin{abstract}
We present periodic nanohole arrays fabricated in free-standing metal-coated nitride films as a platform for trapping and analyzing single organelles. When a microliter-scale droplet containing mitochondria is dispensed above the nanohole array, the combination of evaporation and capillary flow directs individual mitochondria to the nanoholes. Mammalian mitochondria arrays were rapidly formed on chip using this technique without any surface modification steps, microfluidic interconnects or external power sources. The trapped mitochondria were depolarized on chip using an ionophore with results showing that the organelle viability and behavior were preserved during the on-chip assembly process. Fluorescence signal related to mitochondrial membrane potential was obtained from single mitochondria trapped in individual nanoholes revealing statistical differences between the behavior of polarized vs. depolarized mammalian mitochondria. This technique provides a fast and stable route for droplet-based directed localization of organelles-ona-chip with minimal limitations and complexity, as well as promotes integration with other optical or electrochemical detection techniques.
\end{abstract}

\section{Introduction}

Mitochondria play important roles in cell-maintenance, ${ }^{1}$ cell-death, ${ }^{2}$ are linked with the aging process, ${ }^{3}$ diseases like cancer, ${ }^{4}$ and recently were used for bioenergy generation on chip. ${ }^{5}$ Technologies facilitating on-chip bioanalytical analysis of mitochondria are therefore extremely relevant for their improved understanding and derived applications. Existing platforms for on-chip mitochondrial analysis are generally dependent on steps such as surface modification of the chip, ${ }^{6,7}$ microfluidic assemblies or electrical connections, ${ }^{8-12}$

*arriaga@umn.edu, sang@umn.edu.

†These authors contributed equally to this work.

The authors declare no competing financial interest.

Supporting Information

Methods detailing nanohole array chip fabrication, mitochondria sample preparation, imaging, data analysis and other calculations are described. This material is available free of charge via the Internet at http://pubs.acs.org. 
which limit the ease of sample delivery and analysis. Attempts have been made recently to overcome these limitations, utilizing platforms such as PDMS wells or optical fiber bundles for the analysis of yeast-derived mitochondria. ${ }^{13}, 14$ These substrates are still reliant on random mitochondrial attachment with no directional control over localization of these organelles, require surface hydrophilization for organelle capture and have little scope for combination with other analytical techniques. Random distribution of mitochondria as well as staining of PDMS substrates by fluorescent dyes such as JC-1 can result in increased background fluorescence, which interferes with sensitive analysis of these organelles. We propose that substrates based on metallic nanohole arrays fabricated in silicon nitride membranes present significant improvements over existing limitations leading to efficient and stable analysis of complex bioparticles such as organelles.

Metal-coated nanohole arrays ${ }^{15}$ have emerged as a versatile bioanalytical platform having been widely used for analytical sensing including surface plasmon resonance, ${ }^{16}$ surfaceenhanced Raman spectroscopy (SERS), ${ }^{17}$ plasmon-enhanced fluorescence, ${ }^{18}$ and singlemolecule analysis. ${ }^{19}$ They have also been utilized for on-chip detection and analysis of complex bioparticles such as exosomes, ${ }^{20}$ viruses,${ }^{21}$ and virus-like particles. ${ }^{22,}{ }^{23}$ However, these platforms have also primarily relied on non-directional capture of molecules and particles, though some examples have spatially-heterogeneous surface chemistry for selective capture of particles. Nanoholes fabricated in free-standing silicon nitride $\left(\mathrm{Si}_{3} \mathrm{~N}_{4}\right)$ films have been shown to be efficient at directing the flow of solution through them, which can be used for directed trapping of particles suspended in solution. ${ }^{24-28}$ As this trapping methodology works well with aqueous media, it can be readily applied for the capture and array-based analysis of biological particles such as organelles.

In this technical note, we demonstrate that a nanohole-based passive flow-driven technique facilitates directed assembly of mammalian mitochondria arrays with exact positioning of single mitochondria inside individual nanoholes. Single mitochondria analysis can enable us to identify differences within as well as between organelle subpopulations, which is an improvement over analysis of aggregated organelles. ${ }^{9,}{ }^{29}$ Spatial control over the localization of mitochondria can reduce random adsorption of organelles improving the signal-to-noise ratio, facilitating array-like analysis and improving integration with other analytical methods such as SERS. ${ }^{27,} 28$ As the evaporation-driven flow minimizes the limitations imposed on the setup by surface-treatment or external attachment, it can be applied towards the stable analysis of complex bioparticles including other organelles and cells. Furthermore this metallic nanohole array system has the potential to utilize plasmonic or electrochemical methods for label-free organelle analysis. ${ }^{17,30}$

\section{Methods}

Details of chip fabrication, preparation of mammalian mitochondria samples, imaging and data analysis can be found in the supporting information file.

\section{Sample loading}

To capture mammalian mitochondria in nanoholes, $10 \mu \mathrm{L}$ of buffer solution containing mitochondria was added to the backside reservoir of the nanohole array chip. 
The setup was left undisturbed for 10 minutes to direct mitochondria over the nanohole array region and eventually trap them inside nanoholes. To remove untrapped mitochondria from the chip, the reservoir area was washed by addition and suction of $10 \mu \mathrm{L}$ of fresh buffer solution using a micropipette. This step was repeated three times. To confirm the trapping of single mitochondria in the nanoholes, two mitochondria samples were prepared. One sample contained mitochondria tagged with DsRed2 fluorescent protein, whereas the other sample had mitochondria tagged with MitoTracker Green (MTG). Equal volumes of the samples were mixed together before loading on the nanohole array substrate. For the depolarization of mitochondria on chip, $10 \mu \mathrm{L}$ of buffer solution with mitochondria containing JC-1 was added to the nanohole array substrate. Once the mitochondria were trapped, $1 \mu \mathrm{L}$ buffer solution containing valinomycin at a concentration of $20 \mu \mathrm{M}$ was added to the reservoir. After dilution, the actual valinomycin concentration in the reservoir was approximately $2 \mu \mathrm{M}$. The samples were imaged after being exposed to valinomycin for 2 minutes.

\section{Results and Discussion}

Nanohole array chips with a 100-nm-thick suspended nitride membrane were prepared as discussed elsewhere (Figure 1). ${ }^{27}$ Mammalian mitochondria in suspension were added to the etched backside reservoir of the chips, on the silicon-nitride surface. Wetting of the nanoholes is initiated by the silicon-nitride layer (contact angle $\sim 45^{\circ}$ ), and the gold films also have favorable conditions for wetting (contact angle $\sim 70^{\circ}$ ). Contact angle for nonwetting liquids should be greater than $90^{\circ}$. The assembly of mitochondria arrays in nanoholes is driven by evaporation from the open end of the nanoholes (Figure 2a). This process works on the principles of the coffee-stain effect where capillary flow towards pinned edges of a water drop results in concentration of particles. ${ }^{31}$ In this case, the meniscus of water inside the nanoholes undergoes constant evaporation and is replenished by flow of water from the large drop of mitochondrial suspension. ${ }^{27}$ This directed flow drags any particles suspended in the aqueous solution towards the nanoholes, where they are trapped. We believe the adherence of mitochondria to the nanoholes is primarily a result of the trench-like nature of the nanoholes. If the size of the mitochondria is relatively close to the diameter of the holes, they can be "tucked-in" the holes and the washing does not provide sufficient vertical drag to displace them. In fact, previous reports have shown that such tucking-in prevents the removal of particles if the hole diameter and particle size are relatively well-matched, when a squeegee-like surface cleaning procedure is used. ${ }^{32}$ In case the mitochondria are loosely adhered or have size much larger than the holes, their removal during the washing step is more likely. Once assembled, the samples were imaged through the gold-coated side of the nanoholes. An image of a suspended membrane area with four mitochondria arrays can be seen in Figure $2 b$. These mitochondria were tagged with a mitochondrial-selective fluorescent label MitoTracker Green (MTG).

These arrays were formed within minutes simply by the addition of solution on the substrate, enabling rapid trapping and simultaneous fluorescence analysis of many mitochondria in a periodic array. The nanoholes have a diameter of $600 \mathrm{~nm}$ and their depth, which is defined by the thickness of the nitride membrane and the thin metal film, is $300 \mathrm{~nm}$. Typical sizes of isolated mitochondria are about $630 \mathrm{~nm}$, with standard deviation of $180 \mathrm{~nm}$ (Figure S3). We 
chose $600 \mathrm{~nm}$ as a starting point for these experiments since we wanted the holes to be optimally sized to trap mitochondria while reducing the chance of multiple mitochondria cohabiting the same hole. We expected the limited size combined with diluted mitochondria suspension to facilitate trapping and visualization of isolated single mitochondria in the nanoholes. This was tested using two mitochondria samples, each tagged with a different fluorophore. In one sample mitochondria were tagged with DsRed2 fluorescent protein whereas in the other sample mitochondria were tagged with MTG. Equal volumes of the samples were mixed, added to the nanohole array substrate and then imaged. An image showing overlap of the two fluorescence channels for these experiments can be seen in Figure 2c. The arrays were analyzed to record the number of holes with green, red and overlapping colors (Figure S1). Green fluorescence was observed from $63 \%$ of the occupied holes (\# of holes = 51), red from 35\% (\# of holes = 34), whereas overlap of colors was seen in a small fraction of the holes $(2 \%)$ in the arrays (\# of holes $=2$ ).

The relative excess of MTG to DsRed2 mitochondria (63\% vs. $35 \%$ ) can be due to a few factors. First, prior to mixing, the mitochondria concentration in the MTG-tagged sample may have been greater than that of the DsRed2 sample, creating an excess of MTG relative to DsRed2 mitochondria. Second, DsRed2 fluorescence is dependent upon expression of DsRed2 protein, which varies across mitochondria and may not be expressed in some mitochondria. In fact, some of the apparently empty nanoholes in the array may be occupied by mitochondria from the DsRed2 sample that do not express DsRed2 or express it at concentrations below the detection threshold, which would skew the apparent distribution toward an excess of MTG-tagged mitochondria. The variation in red fluorescence intensities visible in Figure 2c can be due to difference in expression of DsRed2 protein amongst the trapped mitochondria. Using the known number of nanoholes and observed number of mitochondria in Figure 2c, we estimated the probability of observing overlaps (calculation in supporting information). We expect to see overlap in 4 out of 500 nanoholes on average, which is close to the observed number.

We demonstrate an application of the organelle-on-a-chip system for fluorescence-based analysis of mitochondrial properties, in particular their membrane potential. The transmembrane potential in mitochondria is an important indicator of function. ${ }^{33}$ Trapped mitochondria were treated with valinomycin that depolarized mitochondria on chip. Valinomycin is a potassium ionophore, which facilitates the transport of potassium ions through the membrane leading to decrease of the mitochondrial membrane potential i.e. depolarization of the membrane. ${ }^{34}$ This change in mitochondrial membrane potential can be visualized using a membrane-permeant JC-1 dye, whose accumulation in the mitochondria is dependent on the transmembrane potential. ${ }^{35}$ The dye can exist in a monomeric form (green fluorescence, emission $\sim 529 \mathrm{~nm}$ ) or can form J-aggregates (red fluorescence, emission $\sim 590 \mathrm{~nm}$ ). Increase in the mitochondrial membrane potential causes accumulation of the dye in the mitochondria, facilitating the increase in the formation of aggregates and leading to higher red-to-green fluorescence ratio. Depolarization of the mitochondrial membrane is indicated by a decrease in ratio of red-to-green fluorescence emission from red $(\sim 590 \mathrm{~nm})$ to green $(\sim 529 \mathrm{~nm})$. For samples under observation, this ratio of red-to-green fluorescence intensity is recorded to monitor the changes in mitochondrial membrane potential. $^{35}$ 
The schematic for two approaches of mitochondria depolarization is shown in Figure 3a. In the first approach valinomycin was added to polarized mitochondria trapped on the chip (Case A), whereas in the second approach mitochondria were depolarized off-chip in bulk suspension (control) and then added to the chip (Case B). These samples were imaged before and after on-chip valinomycin treatment. The JC-1 fluorescence values were monitored and the ratios were calculated. Plots showing the fluorescence ratios before and after on-chip addition of valinomycin for twenty-five holes on a single array are shown in Figure 3b-c. The decrease in the red-to-green fluorescence ratio for each nanohole can be tracked from the plot. The variation in the fluorescence ratio for the polarized samples can be due to in-sample heterogeneity i.e. difference in the transmembrane potential or functional states of different trapped mitochondria. Fluorescence ratios for all nanoholes in a single array are shown in the form of vertical scatter plots (Figure S2). Before valinomycin addition, the mean value of red-to-green fluorescence for the polarized samples was 0.55 and the median was 0.47 . After valinomycin addition, the mean value changed to 0.31 whereas the median changed to 0.30 . For the bulk-depolarized control samples, the mean and median fluorescence ratios before on-chip valinomycin addition were both 1.01, and showed no change after on-chip valinomycin addition. Unpaired t-tests performed for the two cases calculated a statistically significant difference in the means for the polarized samples ( $\mathrm{P} \leq 0.0001)$, whereas for the control samples the change was not significant $(\mathrm{P}=$ $0.1138)$.

The change in the red-to-green fluorescence ratio before and after on-chip addition of valinomycin were calculated. This change is defined as the difference between the fluorescence ratio before and after addition of valinomycin on chip $(\Delta(R / G)=R / G$ before $\mathrm{R} / \mathrm{G}$ after). A scatter plot for calculated $\Delta(\mathrm{R} / \mathrm{G})$ for the nanoholes in a single array for both cases is shown in Figure 3d. The mean change for the polarized samples was about 0.25 units, whereas the change for the bulk-depolarized control samples was close to zero. A ttest comparing the calculated change for a polarized sample vs. control also showed that there is statistically significant difference $(\mathrm{P} \leq 0.0001)$ between the changes in fluorescenceratios observed. The observed response aligns well with our expectations from the samples and confirms that the mitochondria maintain their viability and function during this on-chip assembly process. The setup allows us to monitor individual mitochondria localized at multiple identifiable spots on the same chip with the ability to perform multiple assays.

\section{Concluding Remarks}

We have shown that nanohole arrays fabricated on nitride membranes can be used as a platform for directed, localized trapping of mitochondria by addition of a micro-droplet. The application of this simple, efficient, self-propelling and biocompatible platform was demonstrated through trapping and subsequent array-based analysis of individual mammalian mitochondria. The effect of external chemical stimulus in the form of valinomycin on polarized mammalian mitochondria was recorded and compared to predepolarized mitochondria. The observed difference in their response clearly indicated that the mitochondria maintain their viability and function after the passive flow-driven on-chip assembly process, indicating the potential of this platform for stable analysis of fragile bioparticles including organelles and cells. A limitation of the discussed platform remains 
non-selective trapping of particles, which can be an issue working with complex biological solutions with diverse components. To overcome this problem, samples pre-enriched with desired particles or receptor-labeled nanoholes can be used. Fabrication techniques such as nanoimprinting can be used in the future to obtain large-scale arrays, allowing us to analyze larger sample sizes. ${ }^{28}$ Importantly, this platform can be easily combined with techniques such as SPR imaging, ${ }^{36}$ nanohole-enhanced SERS ${ }^{17,} 28$ or electrochemistry ${ }^{30}$ for highthroughput multimodal organelle-on-a-chip analysis. Hence we believe this platform is a uniquely attractive tool for stable organelle analysis as it promotes directed localization with minimal limitations and complexity, while supporting diverse analytical techniques.

\section{Supplementary Material}

Refer to Web version on PubMed Central for supplementary material.

\section{Acknowledgments}

(The authors thank support by the grants from the National Institutes of Health (NIH R01 GM 092993 for S.K., N.J.W., S.-H.O.; NIH R01 AG020866 for G.G.W. and E.A.; NIH T32 AG029796 for G.G.W.), the National Science Foundation (NSF CAREER Award for S.K., N.J.W., and S.-H.O.), and the MnDRIVE Research Initiative (N.J.W. and S.-H.O.). This work utilized resources at the University of Minnesota, including the Minnesota Nanofabrication Center, which receives partial support from NSF through the National Nanotechnology Infrastructure Network program, and the Characterization Facility, which has received capital equipment funding from NSF through the Materials Research Science and Engineering Center. S.K. acknowledges support from the Doctoral Dissertation Fellowship from the University of Minnesota.

\section{References}

1. Hatefi Y. Annu. Rev. Biochem. 1985; 54:1015-1069. [PubMed: 2862839]

2. Newmeyer DD, Ferguson-Miller S. Cell. 2003; 112:481-490. [PubMed: 12600312]

3. Balaban RS, Nemoto S, Finkel T. Cell. 2005; 120:483-495. [PubMed: 15734681]

4. Fulda S, Galluzzi L, Kroemer G. Nat. Rev. Drug Discov. 2010; 9:447-464. [PubMed: 20467424]

5. Wasylycia JR, Sapelnikova S, Jeong H, Dragoljic J, Marcus SL, Harrison DJ. Lab Chip. 2008; 8:979-982. [PubMed: 18497920]

6. Nakayama S, Sakuyama T, Mitaku S, Ohta Y. Biochem. Biophys. Res. Commun. 2002; 290:23-28. [PubMed: 11779127]

7. Quarato G, Piccoli C, Scrima R, Capitanio N. Mitochondrion. 2011; 11:764-773. [PubMed: 21762790]

8. Johnson RD, Navratil M, Poe BG, Xiong G, Olson KJ, Ahmadzadeh H, Andreyev D, Duffy CF, Arriaga EA. Anal. Bioanal. Chem. 2007; 387:107-118. [PubMed: 16937092]

9. Wolken GG, Kostal V, Arriaga EA. Anal. Chem. 2011; 83:612-618. [PubMed: 21192658]

10. Lim TS, Davila A, Zand K, Wallace DC, Burke PJ. Wafer-scale mitochondrial membrane potential assays. Lab Chip. 2012; 12:2719-2725. [PubMed: 22627274]

11. Zand K, Pham T, Davila A Jr, Wallace DC, Burke PJ. Anal. Chem. 2013; 85:6018-6025. [PubMed: 23678849]

12. Luo J, Abdallah BG, Wolken GG, Arriaga EA, Ros A. Biomicrofluidics. 2014; 8:021801. [PubMed: 24959306]

13. Suraniti E, Vajrala VS, Goudeau B, Bottari SP, Rigoulet M, Devin A, Sojic N, Arbault S. Anal. Chem. 2013; 85:5146-5152. [PubMed: 23600852]

14. Vajrala VS, Suraniti E, Garrigue P, Goudeau B, Rigoulet M, Devin A, Sojic N, Arbault S. Anal. Bioanal. Chem. 2014; 406:931-941. [PubMed: 23892878]

15. Ebbesen TW, Lezec HJ, Ghaemi HF, Thio T, Wolff PA. Nature. 1998; 391:667-669. 
16. Brolo AG, Gordon R, Leathem B, Kavanagh KL. Langmuir. 2004; 20:4813-4815. [PubMed: 15984236]

17. Brolo AG, Arctander E, Gordon R, Leathem B, Kavanagh KL. Nano Letters. 2004; 4:2015-2018.

18. Brolo AG, Kwok SC, Moffitt MG, Gordon R, Riordon J, Kavanagh KL. J. Am. Chem. Soc. 2005; 127:14936-14941. [PubMed: 16231950]

19. Levene MJ, Korlach J, Turner SW, Foquet M, Craighead HG, Webb WW. Science. 2003; 299:682-686. [PubMed: 12560545]

20. Im H, Shao H, Park YI, Peterson VM, Castro CM, Weissleder R, Lee H. Nat. Biotechnol. 2014; 32:490-495. [PubMed: 24752081]

21. Yanik AA, Huang M, Kamohara O, Artar A, Geisbert TW, Connor JH, Altug H. Nano Lett. 2010; 10:4962-4969. [PubMed: 21053965]

22. Jackman JA, Linardy E, Yoo D, Seo J, Ng WB, Klemme DJ, Wittenberg NJ, Oh S-H, Cho NJ. Small. 2015

23. Junesch J, Emilsson G, Xiong K, Kumar S, Sannomiya T, Pace H, Vörös J, Oh S-H, Bally M, Dahlin AB. Nanoscale. 2015; 7:15080-15085. [PubMed: 26351000]

24. Eftekhari F, Escobedo C, Ferreira J, Duan X, Girotto EM, Brolo AG, Gordon R, Sinton D. Anal. Chem. 2009; 81:4308-4311. [PubMed: 19408948]

25. Yanik AA, Huang M, Artar A, Chang T-Y, Altug H. Appl. Phys. Lett. 2010; 96:021101.

26. Escobedo C, Brolo AG, Gordon R, Sinton D. Optofluidic concentration: plasmonic nanostructure as concentrator and sensor. Nano Lett. 2012; 12:1592-1596. [PubMed: 22352888]

27. Kumar S, Wittenberg NJ, Oh S-H. Anal. Chem. 2012; 85:971-977. [PubMed: 23214989]

28. Kumar S, Cherukulappurath S, Johnson TW, Oh S-H. Chem. Mat. 2014; 26:6523-6530.

29. Wolken GG, Arriaga EA. Anal. Chem. 2014; 86:4217-4226. [PubMed: 24673334]

30. Dahlin AB, Sannomiya T, Zahn R, Sotiriou GA, Vörös J. Nano Lett. 2011; 11:1337-1343. [PubMed: 21275409]

31. Deegan RD, Bakajin O, Dupont TF, Huber G, Nagel SR, Witten TA. Nature. 1997; 389:827-829.

32. Wittenberg NJ, Im H, Johnson TW, Xu X, Warrington AE, Rodriguez M, Oh S-H. ACS Nano. 2011; 5:7555-7564. [PubMed: 21842844]

33. Brand MD, Nicholls DG. Biochem. J. 2011; 435:297-312. [PubMed: 21726199]

34. Moore C, Pressman BC. Biochem. Biophys. Res. Commun. 1964; 15:562-567.

35. Smiley ST, Reers M, Mottola-Hartshorn C, Lin M, Chen A, Smith TW, Steele GD, Chen LB. Proc. Natl. Acad. Sci. U. S. A. 1991; 88:3671-3675. [PubMed: 2023917]

36. Lindquist NC, Lesuffleur A, Im H, Oh S-H. Lab Chip. 2009; 9:382-387. [PubMed: 19156286] 

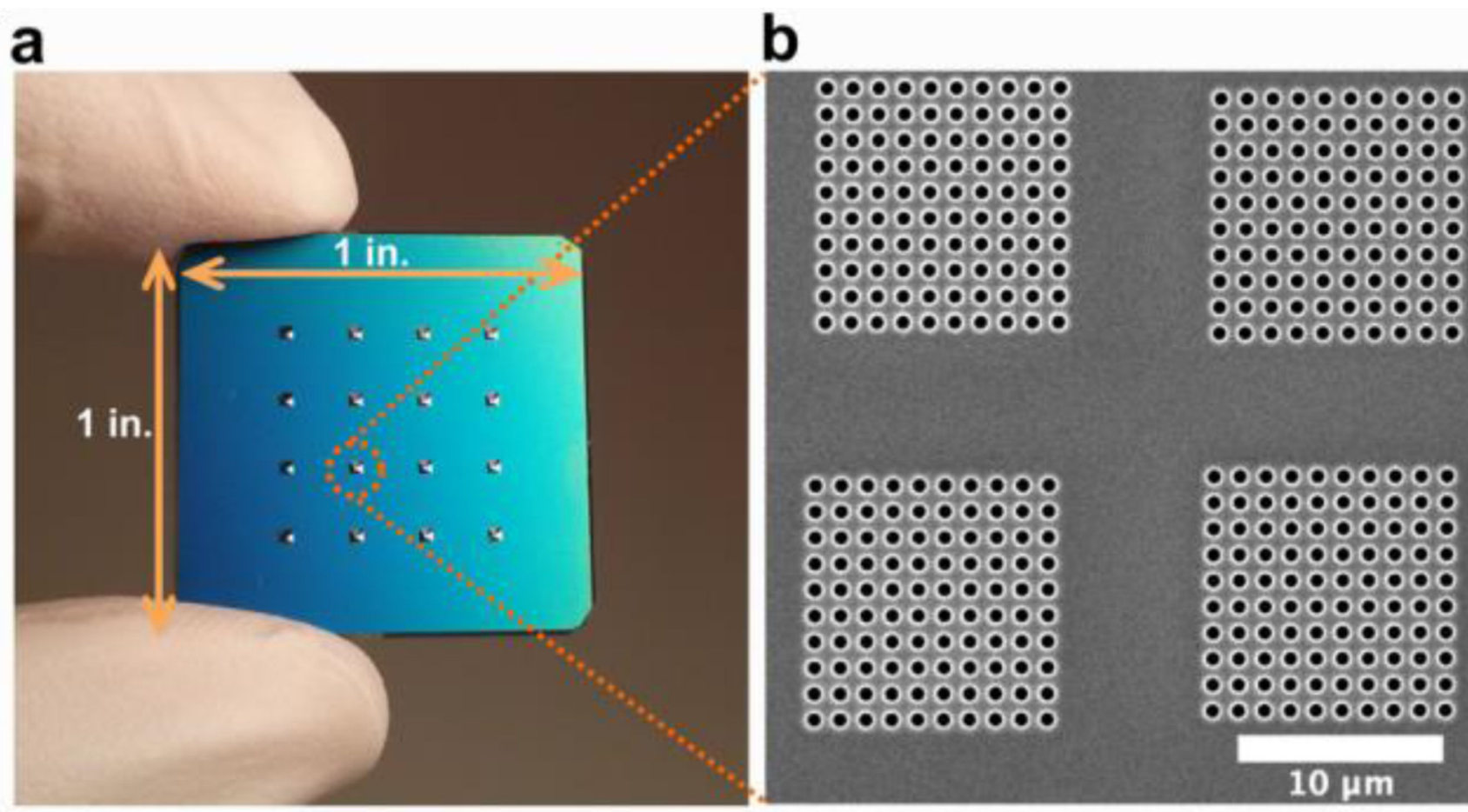
0000000 00000 - $\bullet \bullet \bullet \bullet \bullet \bullet \bullet$ 00000000 000000 0000000 00000000

Figure 1.

(a) A photograph showing the backside of a 1 inch $\times 1$ inch silicon chip with $16 \mathrm{KOH}$ etched reservoirs visible. (b) A SEM showing 4 arrays milled on the suspended nitride membrane with hole size $600 \mathrm{~nm}$ and periodicity $1200 \mathrm{~nm}$. 


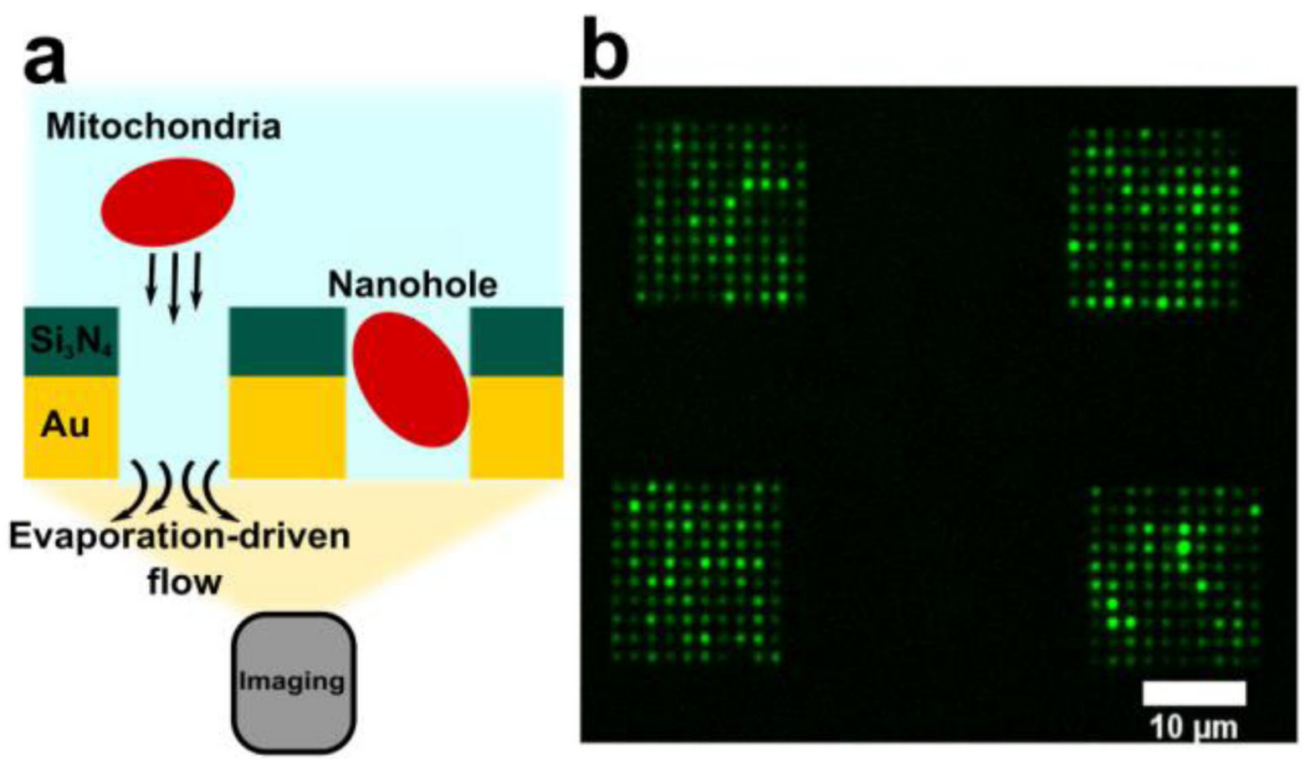

C

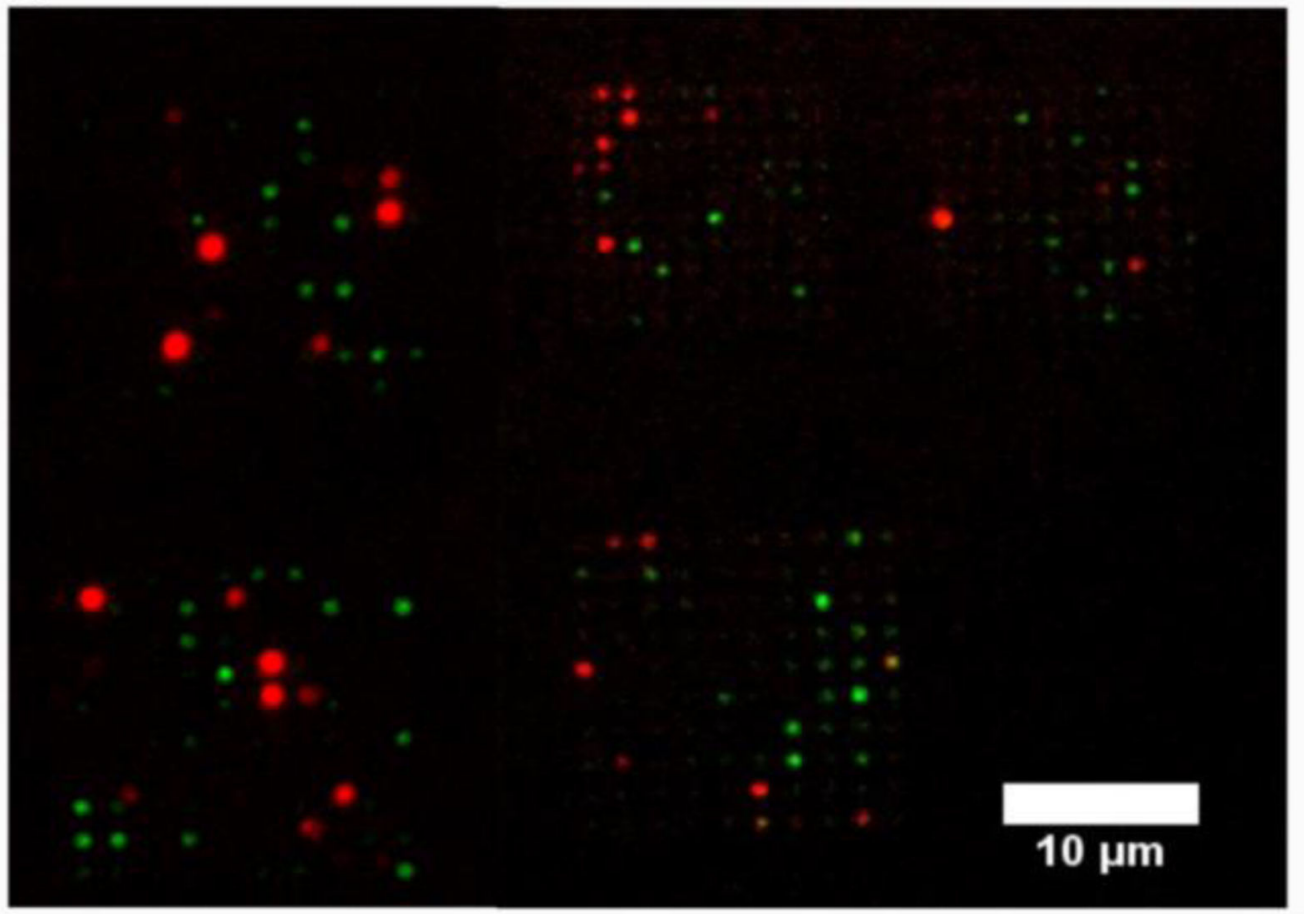

Figure 2.

Sample analysis in an array format. (a) Sample was added to the backside reservoir on the chip and mitochondria were allowed to assemble on to the nanoholes. The samples were washed and then imaged from the gold-coated end of the nanoholes. (b) Image shows 4 arrays containing trapped mitochondria tagged with MitoTracker Green dye. (c) Two samples containing mitochondria tagged with a fluorescent marker (DsRed2 in one sample and MitoTracker Green in the other) were diluted and mixed. The mixed mitochondria suspension was then added to the chip over the nanohole arrays for the capture of 
mitochondria. Image shows 5 arrays containing captured mitochondria tagged with either DsRed2 or MitoTracker Green. 
a
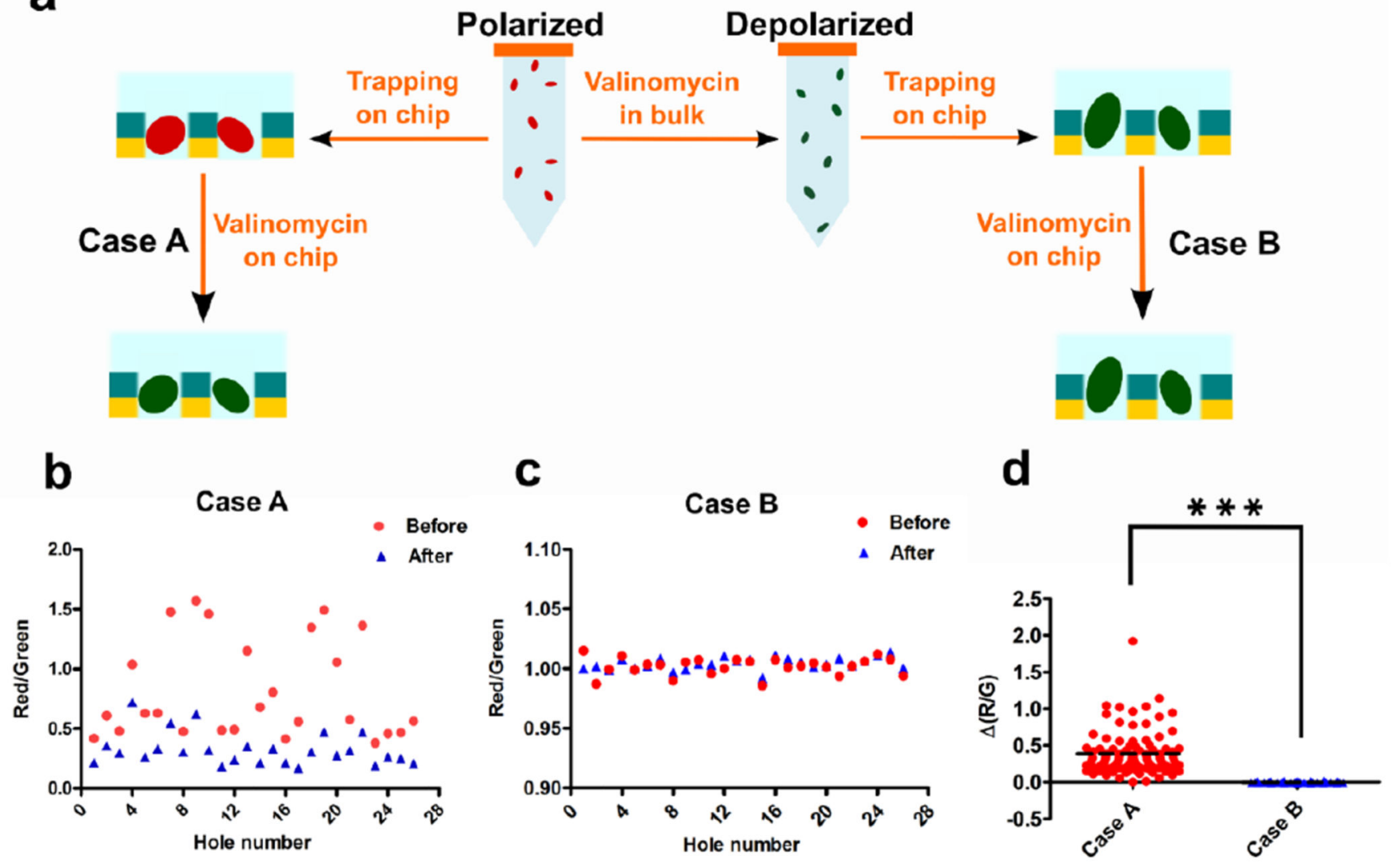

Figure 3.

Mitochondria tagged with JC-1 dye were treated with valinomycin and the change in the ratio of red-to-green fluorescence was monitored for each nanohole. (a) Depiction of the experimental scheme using two samples of mitochondria. On-chip valinomycin addition to polarized mitochondria has been termed as Case A whereas addition of valinomycin to already depolarized mitochondria has been termed as Case B. (b) 25 holes on an array containing polarized mitochondria i.e. case A. Vertically aligned data points were obtained from the same hole before and after on-chip valinomycin addition. (c) 25 holes on an array containing bulk-depolarized mitochondria i.e. case B. Vertically aligned data points were obtained from the same hole before and after on-chip valinomycin addition. (d) The difference in the red-to-green fluorescence ratio (i.e. $\Delta(\mathrm{R} / \mathrm{G})=\mathrm{R} / \mathrm{G}$ before $-\mathrm{R} / \mathrm{G}$ after) shown for an array with polarized mitochondria (case A) and pre-depolarized mitochondria (case B). T-test indicates significant difference in the change observed for the two cases, $* * * p<0.0001$. Dashed line indicates the mean value for all the plots. 